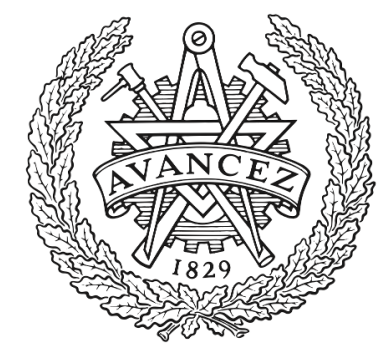

CHALMERS

UNIVERSITY OF TECHNOLOGY

\title{
Process Mining for Quality Improvement: Propositions for Practice and Research
}

Downloaded from: https://research.chalmers.se, 2023-04-26 09:06 UTC

Citation for the original published paper (version of record):

Dahlin, S., Eriksson, H., Raharjo, H. (2019). Process Mining for Quality Improvement: Propositions for Practice and Research. Quality Management in Health Care, 28(1): 8-14. http://dx.doi.org/10.1097/QMH.0000000000000197

N.B. When citing this work, cite the original published paper. 


\title{
PROCESS MINING FOR QUALITY IMPROVEMENT: PROPOSITIONS FOR PRACTICE AND RESEARCH
}

\begin{abstract}
Process mining offers ways to discover patient flow, check how actual processes conform to a standard, and use data to enhance or improve processes. Process mining has been used in healthcare for about a decade, however, with limited focus on quality improvement. Hence, the aim of the paper is to present how process mining can be used to support quality improvement, thereby bridging the gap between process mining and quality improvement. We have analysed current literature to perform a comparison between process mining and process mapping. To better understand how process mining can be used for quality improvement we provide two examples. We have noted four limitations that must be overcome, which have been formulated as propositions for practice. We have also formulated three propositions for future research. In summary, although process mapping is still valuable in quality improvement, we suggest increased focus on process mining. Process mining adds to quality improvement by providing a better understanding of processes in terms of uncovering (un)wanted variations as to obtain better system results.
\end{abstract}

Keywords: Process Mining, Quality Improvement, Digitalisation, Process Mapping, Patient pathways

\section{INTRODUCTION}

Currently, many healthcare organisations use process mapping to understand healthcare processes and patient pathways. This method is useful for showing an overview of healthcare processes, particularly in terms of how inputs are transformed into outputs that provide values for patients and/or customers.[1] However, patients do not follow the same pathway as described in the process map. Not only is there variation across different places across healthcare providers (synchronic variation), but changes occur over time at the same healthcare provider (diachronic variation). The first step toward decreasing variation is understanding it, resulting in decreased costs and quality improvement.[2] This raises the question: How can process variations be understood if only process mapping is used?

The rise of healthcare digitalisation has facilitated new ways of analysing and understanding processes using bottom-up approaches. In contrast to process mapping that is based primarily on information derived from aggregate-level data, process mining utilises information from individual patients. Previous research indicates a need for more robust measurement systems for care transitions to more accurately 'capture patients' journeys across different clinical settings'.[3] In response to this, process mining offers a way of using processes' event log data to replay the history and discover what has occurred, check compliance, analyse bottlenecks, compare process variants, and suggest improvements associated with patient pathways and healthcare processes.[4] Process deviations may also reveal improvement possibilities.[5]

Process mining has been used in healthcare for about a decade;[6] unfortunately, it has had limited focus on quality improvement.[7] Recently, the benefits of process mining in healthcare quality improvement have been identified, albeit with a limited scope.[8] However, the potential does not seem to have reached the broader audience of quality improvement practitioners and researchers. With appropriate software, practitioners and researchers could utilise this important methodology to understand and improve patient pathways. Therefore, in this paper, we advocate for the use of process mining in achieving these goals. The aim is to present how process mining can be used to support quality 
improvement, thereby identifying propositions for research and practice. We regard process mining as a natural complement to process mapping in the shift toward increased healthcare digitalisation.

\section{UNDERSTANDING AND IMPROVING HEALTHCARE PROCESSES}

\section{Healthcare redesign through process mapping}

There are several methodologies and tools available for understanding patient pathways and healthcare processes. An overall framework is healthcare redesign, which aims to understand and redesign patient pathways to reduce care variation and improve public satisfaction.[9] This originates from merging continuous quality improvement and business re-engineering.

Healthcare redesign typically includes process mapping, detailed process analysis, imagining the 'ideal' process, identifying required practical changes, and testing and evaluating the implemented changes. The steps required in healthcare design using process mapping can be summarised as follows: i) mapping the existing as-is care process; ii) analysing where problems exist in the process and questioning each step (e.g. Why is the activity done? By whom? Where? In what sequence? Is there a better way?); iii) imagining the ideal to-be care process; iv) identifying required practical changes for the process to become closer to the ideal and v) testing the implemented changes and evaluate if they lead to improvement. Trebble, et al.[1] present four qualitative ways of healthcare process mapping: multi-disciplinary meetings, walking the journey, direct observation of a patient's journey, and the patient's self-reported experience. The resulting process map provides a comprehensive illustration of the patient's pathway. Analysing problems and identifying practical changes require expert domain knowledge, while imagining the 'ideal' process must also include knowledge about evidence-based practice.

\section{Process mining as a complement to process mapping}

Process mining bridges data mining and process management by extracting process knowledge from event logs.[4] Van Der Aalst, et al.[10] argue that it is an enabling methodology, for example for quality improvement and six sigma. Therefore, it offers a more rigorous compliance check and 'ascertain[s] the validity and reliability of information about an organization's core processes' (p. 172). Event logs consist of ordered data that connect a case (e.g. a patient) to a well-defined activity (e.g. surgery). Additionally, timestamps and additional data can be included. Often, this is how Health Information Systems (HIS) collect patient data.[11] An overview of the main differences between process mapping and process mining is presented in Table 1. 
Table 1. Differences between process mining and process mapping.

\begin{tabular}{|c|c|c|}
\hline & Process Mapping & Process Mining \\
\hline Goal & $\begin{array}{l}\text { Understanding and improvement of } \\
\text { processes }\end{array}$ & $\begin{array}{l}\text { Discovery, monitoring, and improvement of } \\
\text { processes }\end{array}$ \\
\hline Data & $\begin{array}{l}\text { From perceived reality among } \\
\text { staff/patients or observations }\end{array}$ & $\begin{array}{l}\text { From data reported in Health Information } \\
\text { Systems (HIS) }\end{array}$ \\
\hline Nature of the Data & Past (historical) & Past (historical) or real-time \\
\hline Process Description & Static & Dynamic \\
\hline Role of Staff & Mapping and improving & Validating and improving \\
\hline Methodology & Mainly qualitative & Mainly quantitative \\
\hline Framework & Data and processes are separated & Data and processes are combined \\
\hline Experts needed & Quality improvers and healthcare staff & $\begin{array}{l}\text { Data scientists, quality improvers, and } \\
\text { healthcare staff }\end{array}$ \\
\hline Main Advantages & $\begin{array}{l}\text { 1) Participants mapping the process } \\
\text { build a shared understanding of the } \\
\text { pathway, } \\
\text { 2) Improvement opportunities can be } \\
\text { identified while mapping. }\end{array}$ & $\begin{array}{l}\text { 1) Reported data can be compared with } \\
\text { 'standard', } \\
\text { 2) Can identify different paths for different } \\
\text { patient groups and individuals, } \\
\text { 3) Improvement opportunities can be } \\
\text { identified more objectively. }\end{array}$ \\
\hline Main Limitations & $\begin{array}{l}\text { 1) The perceived process map may not } \\
\text { be in accordance with 'reality',[12] } \\
\text { 2) Creates only a static picture,[13] } \\
\text { 3) Is resource-demanding.[14] }\end{array}$ & $\begin{array}{l}\text { 1) Current practitioner knowledge limitations } \\
\text { about use of process mining,[15] } \\
\text { 2) Seldom connected to quality } \\
\text { improvement,[7] } \\
\text { 3) Poor HIS data quality[16] may not be in } \\
\text { accordance with 'reality', } \\
\text { 4) Heterogeneity between elements such as } \\
\text { patients, data granularity, and time stamps } \\
\text { often require data pre-processing.[17] }\end{array}$ \\
\hline
\end{tabular}

Process mining is typically used for three different purposes: i) discovery, ii) conformance, and iii) enhancement.[4] Discovery involves identifying event order, which results in a process map. This map is similar to process mapping, but usually provides significantly more detail than is found in a healthcare setting. Conformance concerns if the reality conforms to a pre-defined to-be process model, such as conformance to a clinical pathway or standard. Enhancement involves using data to develop existing process models that better fit reality or include additional information in the model (e.g. ward names). For these purposes, one can use three different orthogonal perspectives; i) control-flow, ii) performance, and iii) organizational.[18] The control-flow perspective leads to visualisation of end-to-end process models and only requires ordered data. Performance connects the pathways and process model with additional data (e.g. timestamps for identification of bottlenecks) or other attribute data (e.g. costs or selected medical treatments), often known as Key Performance Indicators (KPIs). Organizational identifies collaborations between groups (e.g. physicians or wards).

Although healthcare processes differ substantially from more structured business processes, there are several algorithms that can handle complex healthcare processes.[17, 19] Available software allows for examination ranging from the individual patient level to the systems level, allowing data to be assessed from different angles. For example, ProM[20] is an extremely versatile, open source process mining platform and is most commonly used by researchers; however, several commercial, more user-friendly software exist such as Celonis,[21] Disco,[22] and QPR ProcessAnalyzer.[23] 


\section{TWO EXAMPLES OF PROCESS MINING FOR QUALITY IMPROVEMENT}

To better understand how process mining can be used for quality improvement we have provided two examples.

\section{Understanding diachronic process variations}

The first example is from Seoul National University Bundang Hospital in South Korea and is extracted from a study by Yoo, et al..[24] In this case, the hospital opened a new building in April 2013 to house their relocated clinical neuroscience centre. The process changes aimed to increase collaboration between departments and treatment efficiency for patients suffering from severe, rare, or incurable diseases. The researchers aimed to determine if and how relocation and subsequent changes in work procedures affected the healthcare process for patients. Event data was extracted from electronic health records before and after relocation.

In their analysis, the researchers used several different process mining tools such as process discovery, control flow, dotted charts for performance analysis, most frequent pathway analysis, and time analysis. Figure 1, an illustration derived from Yoo, et al.,[24] shows the results of the most frequent flows identified in the process mined event log from the clinical neuroscience centre. Each box represents one activity in the patient pathway and each line represents a transition between two activities, with thicker lines representing more common transitions. The numbers show the frequency of each activity (within the activity box) and transition (next to line). Red lines illustrate patient transitions before relocation, while green lines illustrate patient transitions resulting from the new hospital environment. Blue lines represent transitions common to both processes. 


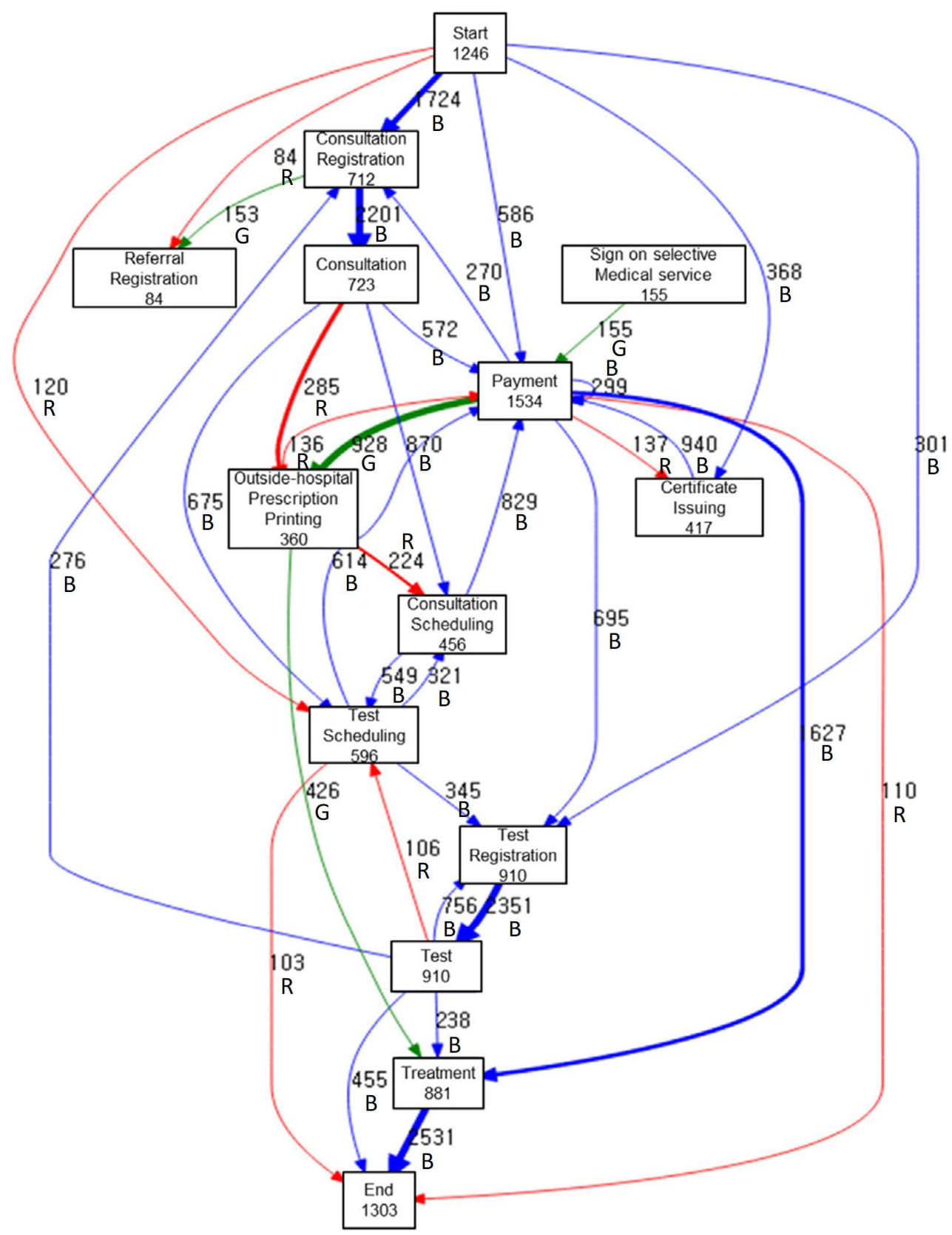

Figure 1: The figure illustrates the patient flow before and after hospital department relocation as described by Yoo et al.[24] Copyright (2016), reprinted with permission from Elsevier. Red lines (R) illustrate transitions before relocation, while green lines $(G)$ illustrate transitions after relocation. Blue lines (B) represent common transitions that occurred prior to and following relocation.

Several changes in the process before and after the relocation were identified. For example, the most common pathway before relocation ( $8.53 \%$ of the patients followed the process 'Certificate issuingpayment-treatment') was replaced by another pathway $(7.67 \%$ of patients followed the process 'Consultation registration-consultation-consultation scheduling-payment-prescription printingtreatment') following relocation (a thorough list of the ten most common pathways prior to and following relocation is presented in Yoo et al.)[24]. Figure 1 complements this list by demonstrating how the new process included less overall variation in transitions of most frequent pathways (i.e. fewer 
green than red lines). Additionally, no remarkable change in overall time spent was identified, although there were slight differences in distribution between activities.

The map, times, and many identified pathway variations help the practitioner understand the system and areas of patient pathway improvement. A key result was the researchers' discovery of discrepancy between the actual flow and the expert-driven process model. Specifically, conformance checking (comparison between the expert-driven model and event log) revealed that the expert-driven model was somewhat less representative of actual pathways within the new establishment and lacked coherence with almost $15 \%$ of patient pathways. This example highlights how process mining can be used to understand diachronic variations of patient pathways and processes (variations over time), rather than studying only KPIs before and after an intervention.

\section{Understanding synchronic process variation}

The next example is derived from Mans, et al.,[25] and highlights variation between two hospitals in the Lombardia Region in Italy and their treatment of stroke patients, from hospital admission to discharge. Micieli, et al.[26] and Quaglini et al.[27] used the same dataset to study compliance to clinical practice and guidelines as well as stroke outcome across hospitals in terms of typical healthcare KPIs (e.g. survival and cost). The researchers used classic statistical analysis. Additionally, Mans, et al. [25] used the dataset to understand how process mining could be used even further to understand between-hospital differences. Based on the Mans, et al.[25] study, Figure 2 displays the treatment processes derived from process mining in two hospitals ( $\mathrm{H} 1$ and $\mathrm{H} 2)$. The numbers in the activity boxes indicate the occurrence frequency of the activity, while the number next to an arrow represents the number of times the activity pattern occurred.
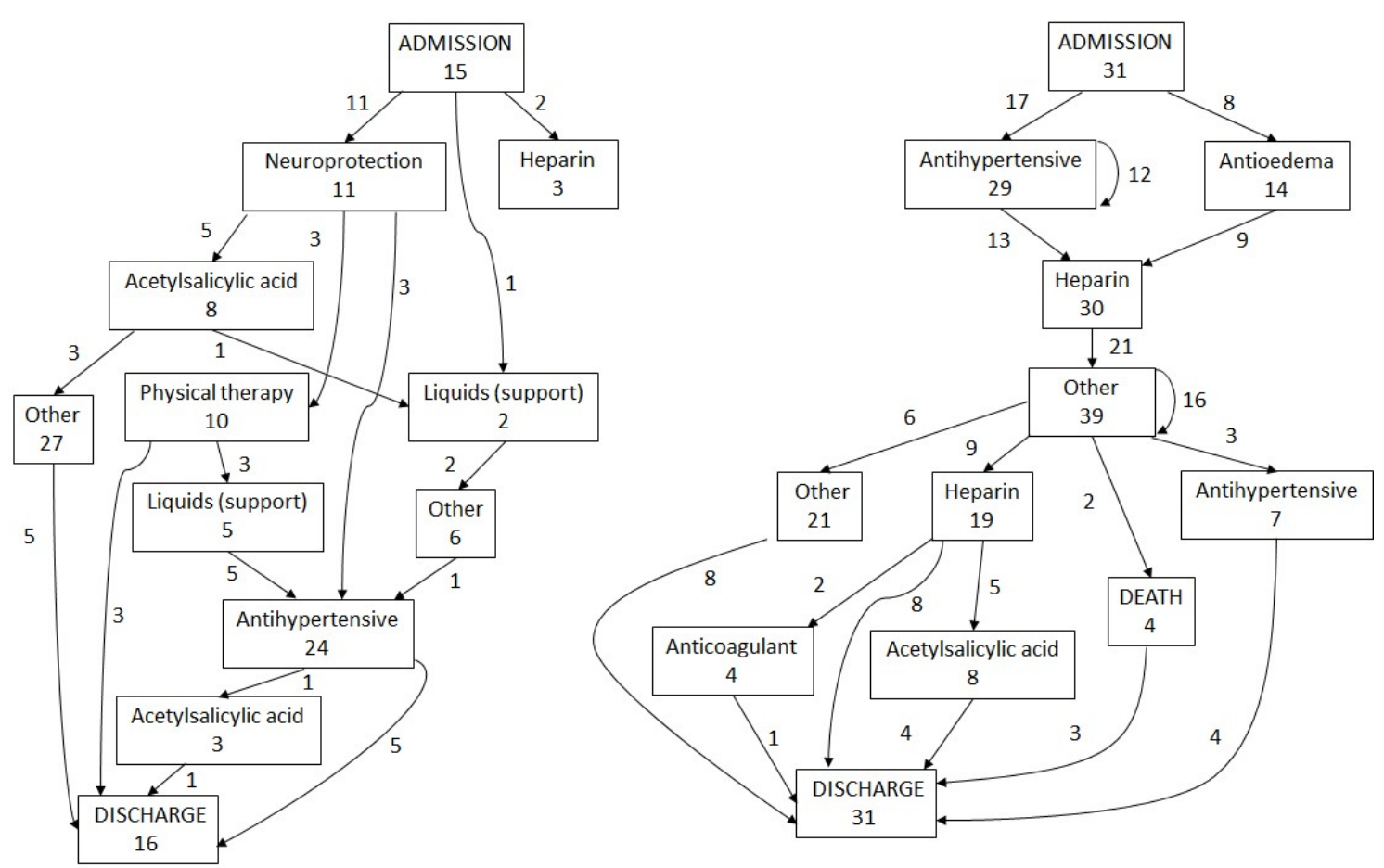

Figure 2: The figure highlights the treatment processes mined for the two hospitals H1(left) and H2 (right). Illustration redrawn and adapted from Mans, et al..[25] Used with permission from EFMI.

Noteworthy differences in patient pathways were discovered (Figure 2). For example, H2 performs hypertension therapy earlier and more frequently than H1. In this paper, antihypertension treatment is 
claimed to be a common practice but not always justified by scientific evidence. Based on the process mining comparison, the authors concluded that $\mathrm{H} 1$ 'adopts therapeutic protocols such as neuroprotection, and also is more compliant with the more recent guidelines, that recommend early physical therapy' (p. 577). This example highlights how synchronic variations in patient pathways and processes can be discovered across healthcare settings with process mining. Synchronic variations are sometimes justified, depending on case mix and context. By displaying process mining maps, it would be possible more easily compare across settings that treat the same patient group. Furthermore, by performing quality improvement that decreases variation, more equal care across settings, regions, and countries could be facilitated.

\section{PROPOSITIONS FOR QUALITY IMPROVEMENT PRACTICE}

There are several models that can be adopted to work with process mining in practice. In 2012, a basic process mining model called the $L^{*}$-life-cycle model was presented.[10] This framework was recently developed to fit the parallel extraction and data analysis from different data sources typically required for healthcare processes.[28] Caron, et al.[29] developed the Clinical Pathway Analysis Model (CPAM), which is a useful model for analysing care pathways consisting of seven phases. Lismont, et al.[17] present a similar model, emphasising the potential need to cluster traces or sequences after perspective selection and reduce complexity as well as iteration between steps. Figure 3 presents a modified CPAM, adapted with the additional clustering step indicated using dotted lines.

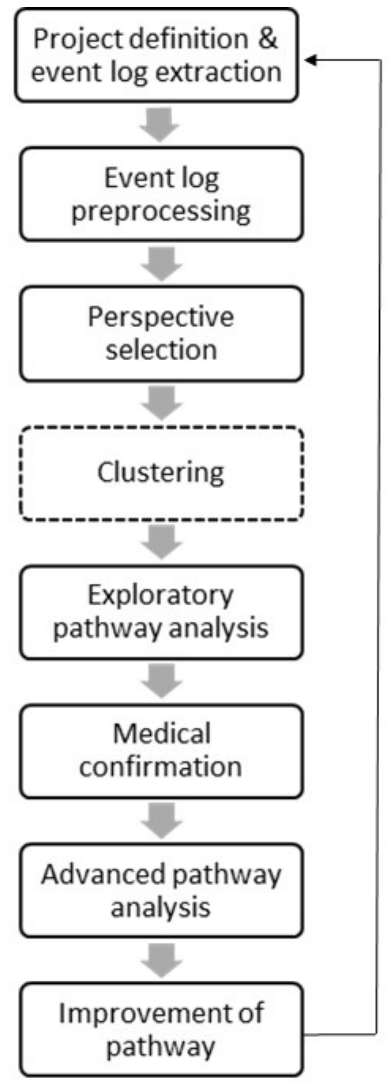

Figure 3: The figure shows an overview with important steps on how process mining can be applied in practice to improve healthcare processes. The figure is a development of the model suggested by Caron, et al.[29] and Lismont, et al..[17] 
Although both types of software and models are available to fully use process mining, it is necessary to overcome limitations in practice. Here, we summarise four propositions, that could provide an overall strategy on how process mining and quality improvement can be integrated and enhanced in healthcare. First, build commitment for and knowledge about process mining[30] (addresses limitation 1 in Table 1) in the organisation, especially among current 'quality improvers'. This can consist of establishing commitment from top management, train experts as well as engage local HIS owners and ICT staff. Second, explore what key healthcare processes that could be driven or evaluated for quality improvement using process mining (follow the suggested steps in Figure 3). Select key health processes that are important for overall results and KPIs, and integrate and use process mining in new or existing quality improvement initiative[24] (addresses limitation 2 in Table 1). Third, be aware of potential challenges (e.g. event correlation, timestamps, snapshots, scoping, granularity) during process mapping[4] and continuously reflect and find ways to improve data quality[16] (addresses limitation 3 in Table 1). Finally, collaborate and confirm with physicians and nurses that what is utilised and discovered in process mining reflects 'reality' [31] (addresses limitation 4 in Table 1) thereby learning and reflecting on the process mining intervention. Subsequently, transfer knowledge within the organisation and explore new key processes where process mining can be used for improvement.

\section{PROPOSITIONS FOR QUALITY IMPROVEMENT RESEARCH}

We also suggest a number of propositions to guide future research about process mining and quality improvement. Whether working with lean, six sigma, value-based healthcare or other concepts, we argue that process mining can be connected to existing concepts and methodologies for an enriched understanding of processes using existing event data stored in the HIS. Therefore, we do not view process mining as a substitute for other methodologies, but rather an expansion of possibilities for more process- and patient-centred care. Specifically, this facilitates integration of different healthcare care processes within and across settings. Although important work has been performed in the field of process mining, few articles describe actual quality improvement.[7] For instance, none refer to root cause analysis, [7] which is central to initiating improvement efforts. In summary, we propose that more empirical research is needed about how process mining can be integrated into quality improvement of patient pathways and healthcare processes.

Healthcare processes and patient pathways remain primarily evaluated only through different KPIs; however, each KPI captures only one aspect of the process.[32, 33] Thus, we argue that researchers should embrace the ways in which the HIS could be an important tool for quality improvement. Subsequently, it is necessary to ensure that the HIS supports a patient and process view and is easy to use for all stakeholders. This requires development of an HIS[25] to reduce errors and limitations and shorten time-consuming data pre-processing.[16, 17, 19, 31] This also includes fool proof documentation of data[34] and coping with implementation challenges.[35] Overall, we propose that research is needed on how the HIS in general, and process mining in particular can be used for developing measurement systems that can be used for quality improvement.

In both process mapping[1] and process mining,[36, 37] collaboration between domain experts from multiple disciplines are of the outmost importance but experts' time can be used differently in each methodology. In process mining, clinical knowledge can be used to identify relevant processes and validate and make clinical sense of mined processes instead of mapping out complex processes. The resulting visualisation has also been reported to enhance understanding and may facilitate more common process understanding during collaboration between different hospital staff,[30] patients and relatives,[25] and individuals outside organisation. Consequently, process mining can support an 
increasingly system-wide perspective on healthcare. One primary challenge for process mining is that if information is not documented in the HIS, it is not discovered during the process. This is an additional reason why collaboration with clinical domain experts is necessary to grasp the real benefits of process mining. Hence, we finally propose that empirical research is needed on how process mining and clinical knowledge can be combined with patient experience to facilitate quality improvement of patient pathways and reality-checks in resource-efficient ways.

\section{CONCLUSIONS}

In this article, we have shed light on the potential of using process mining for quality improvement. As we have learned, every system is perfectly designed to get the results it gets, meaning we need to change the system to improve the results. Therefore, it starts with understanding the system, its processes and their variation. Process mining adds to quality improvement by providing better understanding of process variations that can be decreased and improved to obtain better system results. Although process mapping is valuable in quality improvement, we suggest increased focus on process mining. Process mining offers ways to discover patient flow, check how actual processes conform to a standard, and use data to enhance or improve processes. However, for process mining to be understood and used in healthcare we have noted four limitations that must be overcome, which have been formulated as propositions for practice. Since additional research is required, we have also formulated three propositions for future research. Overall, we urge quality improvement researchers to discover and use process mining. Berwick[38] wrote, 'The ways in which people and organizations try to overcome the destructive forces of entropy in complex systems and to continually improve the work that they do on behalf of patients are numerous and, thank goodness, will forever evolve.' (p. 2093). Our hope is that this paper can facilitate evolution in how quality improvement is approached and be useful not only for healthcare practitioners and researchers in visualising and understanding processes, but also for researchers who aim to identify more effective ways to implement process mining and improve healthcare quality. 


\section{REFERENCES}

1. Trebble TM, Hansi N, Hydes T, et al. Process mapping the patient journey through health care: an introduction. BMJ 2010;341:c4078.

2. Deming, WE. Out of the Crisis. Cambridge, MA: MIT Press 2000.

3. Mountford J, Shojania KG. Refocusing quality measurement to best support quality improvement: local ownership of quality measurement by clinicians. BMJ Qual Saf 2012;21:519-23. doi:10.1136/bmjqs-2012-000859

4. van der Aalst W. Process Mining: Data Science in Action. New York, NY: Springer 2016:3-23.

5. Kahol K, Vankipuram M, Patel VL, et al. Deviations from protocol in a complex trauma environment: errors or innovations? J Biomed Inform 2011;44:425-43. doi:10.1016/j.jbi.2011.04.003 6. Rojas E, Munoz-Gama J, Sepúlveda M, et al. Process mining in healthcare: a literature review. J Biomed Infor 2016;61:224-36. doi:10.1016/j.jbi.2016.04.007

7. Yang W, Su Q. Process mining for clinical pathway: literature review and future directions. Paper presented at the 2014 11th International Conference on Service Systems and Service Management (ICSSSM) 2014.

8. Bergs J, Vandijck D, Hoogmartens $O$, et al. Emergency department crowding: time to shift the paradigm from predicting and controlling to analysing and managing. Int Emerg Nurs 2016;24:74-7. doi:10.1016/j.ienj.2015.05.004

9. Locock L. (2003). Healthcare redesign: meaning, origins and application. Qual Safe Health Care 2003;13:53-7. doi:10.1136/qhc.12.1.53

10. van der Aalst W, Adriansyah A, de Medeiros AKA, et al. Process mining manifesto. Paper presented at the Business Process Management workshops 2012.

11. Coorevits $P$, Sundgren $M$, Klein $G$, et al. Electronic health records: new opportunities for clinical research. J Intern Med 2013;274:547-60. doi: 10.1111/joim.12119

12. Mans RS, van der Aalst WM, Vanwersch RJ, et al. Process mining in healthcare: Data challenges when answering frequently posed questions. In: Lenz R, Miksch S, Peleg $M$, Reichert $M$, Riaño $D$, ten Teije A, eds. Process Support and Knowledge Representation in Health Care. Berlin, Heidelberg: Springer 2013:140-53.

13. Rebuge Á, Ferreira DR. Business process analysis in healthcare environments: a methodology based on process mining. Info Syst 2012;37:99-116. doi:10.1016/j.is.2011.01.003

14. Kim E, Kim S, Song M, et al. Discovery of outpatient care process of a tertiary university hospital using process mining. Healthc Inform Research 2013;19:42-9. doi:10.4258/hir.2013.19.1.42 15. Rovani M, Maggi FM, de Leoni M, et al. Declarative process mining in healthcare. Expert Syst Appl 2015;42:9236-51. doi:10.1016/j.eswa.2015.07.040

16. Suriadi S, Andrews $\mathrm{R}$, ter Hofstede $\mathrm{AH}$, et al. Event log imperfection patterns for process mining: towards a systematic approach to cleaning event logs. Info Syst 2017;64:132-50.

doi:10.1016/j.is.2016.07.011

17. Lismont J, Janssens A.-S, Odnoletkova, I, et al. A guide for the application of analytics on healthcare processes: a dynamic view on patient pathways. Comput Biol Med 2016:77:125-34. doi:10.1016/j.compbiomed.2016.08.007

18. Mans R, Schonenberg M, Song M, et al. Application of process mining in healthcare-a case study in a Dutch hospital Biomed Eng Sys Technol Int Jt Conf BIOSTEC Revis Sel Pap 2009;25:425-38. doi:10.1007/978-3-540-92219-3_32 
19. Mans RS, van der Aalst W, Vanwersch RJ. Process Mining in Healthcare: Evaluating and Exploiting Operational Healthcare Processes. Berlin, Heidelberg: SpringerBriefs in Business Process Management 2015.

20. Process Mining Group. ProM Website. http://www.processmining.org/prom/start. Accessed Oct 19, 2018.

21. Celonis. Celonis solutions Website. http://www.celonis.com/solutions/. Accessed Oct 19, 2018.

22. Fluxicon. Disco software Website. http://www.fluxicon.com/disco/. Accessed Oct 19, 2018.

23. QPR Software Plc. QPR ProcessAnalyzer Website. http://www.qpr.com/products/qprprocessanalyzer, Accessed Oct 19, 2018.

24. Yoo S, Cho M, Kim E, et al. Assessment of hospital processes using a process mining technique: outpatient process analysis at a tertiary hospital. Intl J Med Infor 2016;88:34-43.

doi:10.1016/j.ijmedinf.2015.12.018

25. Mans R, Schonenberg $\mathrm{H}$, Leonardi $\mathrm{G}$, et al. Process mining techniques: an application to stroke care. Stud Health Technol Inform 2008;136:573-8.

26. Micieli G, Cavallini A, Quaglini S. Guideline Application for Decision Making in Ischemic Stroke (GLADIS) Study Group. Guideline compliance improves stroke outcome: A preliminary study in 4 districts in the Italian region of Lombardia. Stroke 2002;33:1341-47.

doi:10.1161/01.STR.0000013663.27776.DB

27. Quaglini S, Cavallini A, Gerzeli S, et al. Economic benefit from clinical practice guideline compliance in stroke patient management. Health Policy 2004;69:305-15.

doi:10.1016/j.healthpol.2003.12.015

28. Helm E, Küng J. Process mining: towards comparability of healthcare processes. Paper presented at the International Conference on Information Technology in Bio-and Medical Informatics; 2016. 29. Caron $\mathrm{F}$, Vanthienen J, Vanhaecht $\mathrm{K}$, et al. A process mining-based investigation of adverse events in care processes. HIM J 2014;43:16-25. doi:10.12826/18333575.2013.0013

30. Helmering $P$, Harrison $P$, lyer $V$, et al. Process mining of clinical workflows for quality and process improvement. Paper presented at the Healthcare Information and Management Systems Society (HIMSS) conference proceedings 2012.

31. Partington A, Wynn M, Suriadi S, et al. Process mining for clinical processes: a comparative analysis of four Australian hospitals. ACM Trans Manag Inf Syst 2015;5:1-19. doi:10.1145/2629446

32. Scobie S, Thomson R, McNeil JJ, et al. Measurement of the safety and quality of health care. Med J Aust 2006;184(Suppl 10):S51.

33. Elg M, Palmberg Broryd K, Kollberg B. Performance measurement to drive improvements in healthcare practice. Int J Oper Prod Manag 2013;33:1623-51. doi:10.1108/IJOPM-07-2010-0208 34. Lanzola G, Parimbelli E, Micieli G, et al. Data quality and completeness in a web stroke registry as the basis for data and process mining. J Healthc Eng 2014;5:163-84. doi:10.1260/2040-2295.5.2.163 35. Gooch P, Roudsari A. Computerization of workflows, guidelines, and care pathways: a review of implementation challenges for process-oriented health information systems. JAMIA 2011;18:73848. doi:10.1136/amiajnl-2010-000033

36. Cho M, Song M, Yoo S. A systematic methodology for outpatient process analysis based on process mining. Paper presented at the Asia-Pacific Conference on Business Process Management 2014.

37. Perimal-Lewis $\mathrm{L}$, De Vries $\mathrm{D}$, Thompson $\mathrm{CH}$. Health intelligence: discovering the process model using process mining by constructing start-to-end patient journeys. Paper presented at the 
Proceedings of the Seventh Australasian Workshop on Health Informatics and Knowledge Management; 2014;153.

38. Berwick DM. (2012). The question of improvement. JAMA 2012;307:2093-94.

doi:10.1001/jama.2012.4146 